\title{
AMENDMENTS
}

\section{Author Correction: Genetics of 35 blood and urine biomarkers in the UK Biobank}

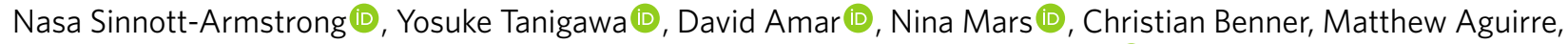
Guhan Ram Venkataraman, Michael Wainberg, Hanna M. Ollila, Tuomo Kiiskinen (D), Aki S. Havulinna,

James P. Pirruccello(D), Junyang Qian, Anna Shcherbina, FinnGen*, Fatima Rodriguez, Themistocles L. Assimes (D), Vineeta Agarwala, Robert Tibshirani, Trevor Hastie, Samuli Ripatti@, Jonathan K. Pritchard (D), Mark J. Daly and Manuel A. Rivas (D)

Correction to: Nature Genetics https://doi.org/10.1038/s41588-020-00757-z, published online 18 January 2021.

In the version of this Article initially published, an error appeared in the third sentence of the Abstract. The sentence originally beginning "We identify 1,857 loci associated with at least one trait" has been modified to read, "We identify 5,794 independent loci associated with at least one trait $\left(p<5 \times 10^{-9}\right)$." In the center panel of Fig. 1, under the "Assessing genetic association" header, "GWAS" subsection, the text "5,792 independent loci" has been corrected to "5,794 independent loci." Further, in Supplementary Table 4a, in the "Number of associations" section, the total for column O, "Number of independent associated loci," the total, originally listed as "5,792," has been corrected to " $5,794 . "$

The errors have been corrected in the online version of the Article.

${ }^{*}$ A list of authors and their affiliations appears online.

Published online: 4 October 2021

https://doi.org/10.1038/s41588-021-00956-2

(c) This is a U.S. government work and not under copyright protection in the U.S.; foreign copyright protection may apply 20212021

\section{Publisher Correction: Epigenomic contributions to tumor cell heterogeneity and plasticity}

Radhika Mathur and Joseph F. Costello (D)

Correction to: Nature Genetics https://doi.org/10.1038/s41588-021-00932-w, published online 4 October 2021.

In the version of this News \& Views initially published, there was a typo in the fifth sentence of the paragraph headed "State shifting and stress response." Specifically, in the sentence now reading "The paradigm of therapeutically targeting glioma stem cells thus has a different relevance in IDH-mutant than in IDH-wild-type gliomas, as the plasticity of the latter necessitates targeting the totality of malignant cells and/or the ability of cells to de-differentiate," the term "plasticity of the former" has been replaced with "plasticity of the latter."

The changes have been made to the online version of the article.

Published online: 20 October 2021

https://doi.org/10.1038/s41588-021-00966-0

๑) Springer Nature America, Inc. 2021 